F. S. Cater, Department of Mathematics, Portland State University, Portland, Oregon 97207, USA

\title{
ON ORDER TOPOLOGIES AND THE REAL LINE
}

\begin{abstract}
We find order topologies that are universal for certain topological properties. An order topology $T$ enjoys a given property if and only if there is an order preserving homeomorphism of $T$ into the universal space for this property. We give similar results for order preserving mappings in place of homeomorphisms.
\end{abstract}

Throughout this note, $T$ will be a linearly ordered set. By the order topology on $T[\mathrm{~K}, 1 \mathrm{I}]$ we mean the topology generated by all intervals of the form $\{x \in T: x<a\}$ and $\{x \in T: x>a\}$ where $a \in T$. We will call this the order space $T$, or the open interval space $T$.

We desire to find an order space $S$ that is separable (has a countable dense subset) such that any order space $T$ is separable if and only if $T$ is homeomorphic to a subspace of $S$. In fact we do not succeed, but we do the next best thing. We find a topology $T^{*}$ on the set $T$, closely associated with the order topology and containing the order topology on $T$, such that the order space $T$ is separable if and only if $T^{*}$ is homeomorphic to a subspace of an appropriate separable order space $S$.

Let $\mathbb{R}$ denote the real line. Give the product $\mathbb{R} \times\{0,1\}$ the dictionary order: $(a, n)<(b, m)$ if and only if $a<b$ in $\mathbb{R}$ or $a=b$ and $n=0, m=1$. We call the resulting order space $\mathbb{R}_{0}$. Note that every subspace of $\mathbb{R}_{0}$ is disconnected. Thus $\mathbb{R}$ is not homeomorphic to any subspace of $\mathbb{R}_{0}$, even though $\mathbb{R}$ is obviously order isomorphic to a subset of $\mathbb{R}_{0}$.

By the enhanced order space $T^{*}$ we mean the topology on the set $T$ generated by all intervals of the form $\{x \in T: x<a\}$ and $\{x \in T: x>a\}$ for any $a \in T$, and of the form $\{x \in T: x \leq b\}$ where $b \in T$ is an element that has no immediate predecessor. Thus the enhanced order topology contains the order topology on $T$.

Key Words: real line, order topology, separable, second countable.

Mathematical Reviews subject classification: 26A03, 26A15, 54A05, 54F05.

Received by the editors November 9, 1999 
Now $\mathbb{R}^{*}$ is commonly called the half open interval space [K, page 59$]$ or the Sorgenfrey line. Moreover, $\mathbb{R}^{*}$ is separable; consider the set of rational numbers.

The enhanced order space $T^{*}$ is the same as the order space $T$ if each element of $T$ has an immediate predecessor or an immediate successor. Thus the spaces $\mathbb{R}_{0}^{*}$ and $\mathbb{R}_{0}$ are the same. Moreover, $\mathbb{R}_{0}$ is separable; consider the set $\{(r, 0): r$ rational $\}$. It is easy to see that $\mathbb{R}^{*}$ is homeomorphic to a subspace of $\mathbb{R}_{0}$.

We will prove that $T$ is a separable order space if and only if $T^{*}$ is homeomorphic to a subspace of $\mathbb{R}_{0}$ (Theorem III).

It will be easier to prove that $T$ is second countable (has a countable basis) if and only if $T$ is homeomorphic to a subspace of $\mathbb{R}$ (Theorem II).

It will be almost trivial to prove that $T$ is countable if and only if $T$ is homeomorphic to a subspace of $\mathbb{Q}$, the space of rational numbers (Theorem I).

Let $T$ be countable. Then the order space $T$ is obviously a second countable regular space and is metrizable by Urysohn's metrization Theorem [K, p. 125]. Then $T$ is homeomorphic to a subspace of $\mathbb{Q}$ by [S, p. 107].

Observe that an analogous argument proves that $T^{*}$ is homeomorphic to a subspace of $\mathbb{Q}$ if $T$ is countable.

To sum up; $\mathbb{R}_{0}$ is our prototype of a separable order space, $\mathbb{R}$ is our prototype of a second countable order space, and $\mathbb{Q}$ is our prototype of a countable order space.

\section{Construction}

Let $E$ be a countable linearly ordered set. Adjoin to $E$ points $-\infty$ and $\infty$ where $-\infty$ is less than any element and $\infty$ is greater than any element of $E$.

We will construct a mapping $f$ of $E \cup\{-\infty, \infty\}$ into $\mathbb{Q}$ as follows. Enumerate $E=\left\{e_{1}, e_{2}, e_{3}, \ldots\right\}$. Let $f(-\infty)=-1$ and $f(\infty)=1, f\left(e_{1}\right)=0$. We define $f\left(e_{n}\right)$ by induction on $n$. Let $f$ be an order preserving mapping of $\{-\infty, \infty\} \cup\left\{e_{1}, \ldots, e_{n-1}\right\}$ into $\mathbb{Q}$. Make $f\left(e_{n}\right)=(f(a)+f(b)) / 2$ where $e_{n} \in$ $(a, b)$ and $a$ and $b$ are consecutive points of the set $\{-\infty, \infty\} \cup\left\{e_{1}, \ldots, e_{n-1}\right\}$. It follows that $f$ preserves order on $\{-\infty, \infty\} \cup\left\{e_{1}, \ldots, e_{n-1}, e_{n}\right\}$. By induction we see that $f$ is an order preserving mapping of $E$ into $Q$.

Lemma 1. Let $(c, d)$ be a bounded complementary interval of the closure of $f(E)$. Then $c \in f(E)$ and $d \in f(E)$.

Proof. Assume $c \notin f(E)$. Choose $c_{1}$ and $d_{1}$ in $f(E)$ such that $c_{1}<c<$ $d \leq d_{1}$ and $d_{1}-c_{1}<2(d-c)$. The interval $\left(c_{1}, d_{1}\right)$ contains infinitely many points of $f(E)$. It follows from the construction that some $e \in E$ satisfies 
$f(e)=\left(c_{1}+d_{1}\right) / 2$. Clearly $\left(c_{1}+d_{1}\right) / 2 \in(c, d)$, contrary to hypothesis. Thus $c \in f(E)$. Similarly $d \in f(E)$.

In what follows, the symbol ${ }^{-}$will denote closure in the Euclidean topology.

Lemma 2. Let $c \in f(E)^{-}$and let $u$ be a real number with $\sup f(E) \geq u>c$. Then there is an $e \in E$ with $f(E)>c$ and

$$
\left\{t \in f(E)^{-}: t<f(e)\right\} \subset\left\{t \in f(E)^{-}: t<u\right\} .
$$

Proof. If $f(E) \cap(c, u)$ is nonvoid, just make $f(e) \in f(E) \cap(c, u)$. So we assume $f(E) \cap(c, u)$ is void. Then there is a $d \in f(E)^{-}$such that $u \in(c, d)$ and $(c, d)$ is a finite complementary interval of $f(E)^{-}$. By Lemma $1, d \in f(E)$. Then $f(e)=d$ suffices.

When $c=\sup f(E)$, Lemma 2 will not be needed in what follows.

Lemma $2^{\prime}$. Let $c \in f(E)^{-}$and let $u$ be a real number with $\inf f(E) \leq u<c$. Then there is an $e \in E$ with $f(e)<c$ and

$$
\left\{t \in f(E)^{-}: t>f(e)\right\} \subset\left\{t \in f(E)^{-}: t>u\right\} .
$$

The proof is like that of Lemma 2 with the inequalities reversed, so we omit it.

Theorem I. Let $T$ be an order space. Then the following are equivalent.

(1) $T$ is countable,

(2) there is an order preserving homeomorphism from $T$ into $Q$,

(3) $T$ is homeomorphic to a subspace of $Q$,

(4) $T$ is order isomorphic to a subset of $Q$.

Proof. (1) $\Rightarrow(2)$ Let $f$ be the order preserving mapping of $T$ into $Q$ from the construction (with $T$ in place of $E$ ). The subspace topology on $f(T)$ in $Q$ contains the order topology on $f(T)$. Let $x \in T$ and $f(x)$ lie in a subbasic open set of one of the forms $\{w \in f(T): w>a\}$ or $\{w \in f(T): w<a\}$ in the subspace topology on $f(T)$. We deduce from Lemmas 2 and $2^{\prime}$ that this set contains an open set in the order topology on $f(T)$ containing $f(x)$. It follows that any open set in the subspace topology on $f(T)$ is an open set in the order topology on $f(T)$. Hence $f$ is a homeomorphism.

Finally, the implications $(1) \Longleftrightarrow(2),(1) \Longleftrightarrow(3),(1) \Longleftrightarrow(4)$ are clear. 
Lemma 3. $T$ is second countable if and only if $T$ is separable and there are at most countably many immediate predecessors (successors) in $T$.

Proof. Suppose $T$ is second countable. Then $T$ is separable. Let $\left\{U_{n}\right\}$ be a countable basis of $T$. For each immediate predecessor $a$, there is an open set $V_{a} \in\left\{U_{n}\right\}$ such that $a$ is the greatest element of $V_{a}$. Thus if $a_{1}$ is another immediate predecessor in $T$, distinct from $a$, then $V_{a} \neq V_{a_{1}}$. It follows that there are countably many immediate predecessors in $T$.

Now suppose that the order space $T$ is separable and there are countably many immediate predecessors in $T$. Let $E$ be a countable subset of $T$ that is dense and includes all the immediate predecessors and successors in $T$. The proof that the countable family of intervals $\left\{\left(e, e^{\prime}\right): e \in E, e^{\prime} \in E\right\}$ is a basis of $T$ is routine, so we leave it.

Theorem II. Let $T$ be an order space. Then the following are equivalent.

(1) $T$ is second countable.

(2) there is an order preserving homeomorphism of $T$ into $\mathbb{R}$.

(3) $T$ is homeomorphic to a subspace of $\mathbb{R}$.

(4) $T$ is order isomorphic to a subset of $\mathbb{R}$.

Proof. (1) $\Rightarrow(2)$ Let $T$ be second countable. By Lemma 3, there is a countable dense subset $E$ of $T$ that contains all the immediate predecessors and successors in $T$ and also the first and last elements of $T$ if any. Let $f$ be the mapping in the construction. For each $x \in T \backslash E$ let

$$
A_{x}=\{t \in E: t>x\} \text { and } B_{x}=\{t \in E: t<x\} .
$$

Then $E=A_{x} \cup B_{x}$.

If $x \in T \backslash E$ and $y \in T \backslash E$ and $x<y$, we claim that $f\left(A_{x}\right) \neq f\left(A_{y}\right)$; for if $f\left(A_{x}\right)=f\left(A_{y}\right)$, then $A_{x}=A_{y}$ and the interval $(x, y)$ could contain no element of $E$ or of $T$, and hence by Lemma 1, $x \in E, y \in E$. Likewise $f\left(B_{x}\right) \neq f\left(B_{y}\right)$.

If $x \in T \backslash E$ we claim that the distance between the sets $f\left(A_{x}\right)$ and $f\left(B_{x}\right)$ is zero; for otherwise, $f\left(A_{x}\right)$ would have a least element $d, f\left(B_{x}\right)$ would have a greatest element $c$ by Lemma 1 , and hence the singleton set $\{x\}$ would be open in $T$ and $x \in E$.

If $x \in T \backslash E$, we claim that $f\left(B_{x}\right)$ has no greatest element; for if $c$ is the greatest element of $f\left(B_{x}\right)$, then the interval $\left(f^{-1}(c), x\right)$ could contain no element of $E$ or of $T$ and consequently $x$ would be an immediate successor and $x \in E$. Likewise $f\left(A_{x}\right)$ has no least element. 
We extend $f$ to a function $g$ from $T$ into $\mathbb{R}$ as follows; If $e \in E$, put $g(e)=f(e)$, and if $x \in T \backslash E$, let $g(x)$ be the real number so that $g(x)>f(e)$ for $e \in B_{x}$ and $g(x)<f(e)$ for $e \in A_{x}$. It follows that $g$ is an order preserving mapping of $T$ into $\mathbb{R}$.

The subspace topology contains the order topology on $g(T)$. From Lemmas 2 and $2^{\prime}$ we deduce that these topologies are in fact the same on $g(T)$. Thus $g$ is an order preserving homeomorphism of $T$ into $\mathbb{R}$.

$(4) \Rightarrow(1)$ Let $S$ be a subset of $\mathbb{R}$. The subspace topology on $S$ is at least as fine as the order topology on $S$. But $\mathbb{R}$ is second countable, so the subspace $S$ is second countable and separable. It follows that the order space $S$ is separable. Finally, $S$ contains at most countably many immediate successors and predecessors, because every family of mutually disjoint intervals in $\mathbb{R}$ is countable. By Lemma 3, the order space $S$ is second countable.

Now the implications $(1) \Longleftrightarrow(2),(1) \Longleftrightarrow(3),(1) \Longleftrightarrow(4)$ are clear.

Before we tackle our last theorem we need an observation about the order space $\mathbb{R}_{0}$.

Lemma 4. Let $S$ be a subset of $\mathbb{R}_{0}$. Then the order space $S$ is separable and the subspace $S$ of $\mathbb{R}_{0}$ is separable.

Proof. Put $A=\left\{a \in \mathbb{R}\right.$ : either $(a, 0)$ or $(a, 1)$ is an isolated point of $S$ in $\left.\mathbb{R}_{0}\right\}$. We claim that $A$ is countable; for otherwise there would be an $a_{0} \in A$ that is both a left and a right accumulation point of $A$ in $\mathbb{R}$, and hence $\left(a_{0}, 0\right)$ would be a left accumulation point of $S$ and $\left(a_{0}, 1\right)$ would be a right accumulation point of $S$ in $\mathbb{R}_{0}$. Thus there are at most countably many isolated points of $S$. Let $J$ denote the set of isolated points in $S$.

For any rational numbers $r_{1}<r_{2}$, select an element $s\left(r_{1}, r_{2}\right)$ in $S$ in the open interval from $\left(r_{1}, 0\right)$ to $\left(r_{2}, 0\right)$ if there is one. We obtain a countable family $P \subset S$ of elements of the form $s\left(r_{1}, r_{2}\right)$. Then $P \cup J$ is a countable subset of $S$, and routine arguments prove that $P \cup J$ is dense in the subspace $S$. Thus the subspace $S$ is separable. But the subspace topology contains the order topology on $S$. It follows that the order space $S$ is also separable.

Theorem III. Let $T$ be an order space. Then the following are equivalent.

(1) $T$ is separable,

(2) there is an order preserving homeomorphism $h$ of $T^{*}$ into $\mathbb{R}_{0}$,

(3) $T^{*}$ is homeomorphic to a subspace of $\mathbb{R}_{0}$,

(4) $T$ is order isomorphic to a subset of $\mathbb{R}_{0}$, 
(5) $T^{*}$ is separable.

Proof. (1) $\Rightarrow(2)$ Let $T$ be separable. Say $E_{0}$ is a countable dense subset of $T$. Let $E=\left\{x \in T: x \in E_{0}\right.$ or $x$ is an immediate successor or predecessor of an element of $\left.E_{0}\right\}$. Then $E$ is a countable dense subset of $T$. Note that if $x$ is the immediate predecessor of $y \in E \backslash E_{0}$, then either $y$ is the immediate successor of a member of $E_{0}$ and $x \in E_{0}$, or $y$ is the immediate predecessor of a member of $E_{0}$ and $y$ is an isolated point necessarily in $E_{0}$ contrary to assumption. Thus the immediate predecessor of a member of $E$ must lie in $E$. Likewise the immediate successor of a member of $E$ must lie in $E$. Put $T_{1}=\{t \in T: t$ has an immediate predecessor but no immediate successor in $T\}$, and $T_{2}=T \backslash T_{1}$.

Observe that if $y \in T_{1} \cap E$ and $x$ is the immediate predecessor of $y$, then $x \in T_{2} \cap E$. Thus if $u<v<w<z$ and $u, v, w, z \in T_{2}$, then the set $(u, w) \cap E$ contains an element $e$ and either $e \in T_{2} \cap E$, or $e \in T_{1} \cap E$ and the immediate predecessor of $e$ lies in $T_{2} \cap E$. It follows that the interval $[u, w)$ meets $T_{2} \cap E$. Likewise $[v, z)$ and $(u, z)$ meet $T_{2} \cap E$. We deduce from this that any open interval $(u, w)$ with $u \in T_{2}, w \in T_{2}$ that meets $T_{2}$ but not $T_{2} \cap E$ has $u \in T_{2} \cap E$. Moreover, any two such intervals cannot have the same left endpoint. But $T_{2} \cap E$ is countable, so there are at most countably many such intervals. It follows that the order space $T_{2}$ is separable.

Now let $(s, t)$ be an open interval with $s \in T_{2}, t \in T_{2}$ such that the open interval $(s, t)$ does not meet $T_{2}$. Then either $(s, t)$ meets $T$ and we have $s \in T_{2} \cap E$ by the argument in the preceding paragraph, or $t$ is the immediate successor of $s$ and $t$ must be an isolated point necessarily in $T_{2} \cap E$. In any case, the closed interval $[s, t]$ meets $T_{2} \cap E$. But $E$ is countable so there are at most countably many immediate successors and predecessors in the order space $T_{2}$. By Lemma 3 the order space $T_{2}$ is second countable.

Let $g$ be the order preserving homeomorphism from $T_{2}$ into $\mathbb{R}$ defined precisely as in the proof of $(1) \Rightarrow(2)$ for Theorem II. Put $h(x)=(g(x), 0) \in \mathbb{R}_{0}$ for $x \in T_{2}$. Observe that if $y \in T_{1}$, then $y$ has an immediate predecessor that is necessarily in $T_{2}$. For $y \in T_{1}$, put $h(y)=(g(x), 1) \in \mathbb{R}_{0}$ where $x$ is the immediate predecessor of $y$. It follows that $h$ is an order preserving mapping of $T$ into $\mathbb{R}_{0}$. It remains to prove that $h$ is a homeomorphism from $T^{*}$ into $\mathbb{R}_{0}$. This will be deduced from considerations of the following four situations.

(i) $x \in T$ and $x$ has an immediate successor and an immediate predecessor in $T$. Then $h(x)$ has an immediate successor and an immediate predecessor in $h(T)$. It follows that the singleton set $\{h(x)\}$ is open in the enhanced order topology of $h(T)$ and open in the subspace topology of $h(T)$.

(ii) $x \in T$ has an immediate predecessor $y$, but no immediate successor, and $x$ is not the greatest element in $T$. It follows that $x \in T_{1}$ and $y \in T_{2}$. It 
also follows that $y$ has no immediate successor relative to the set $T_{2}$ (observe that any element of $T_{1}$ exceeding $x$ is the immediate successor of another element of $T_{2}$ exceeding $x$ ). We deduce that the distance (in $\mathbb{R}$ ) between $g(y)$ and the set $\left\{g(t): t \in T_{2}, t>y\right\}$ is zero; otherwise $\inf \left\{g(t): t \in T_{2}, t>y\right\}$ would be the immediate successor of $g(y)$ in $g\left(T_{2}\right)$ by Lemma 1 , and $y$ would have an immediate successor in $T_{2}$. From this it follows that the set $\{W \in$ $h(T) W \leq h(x)\}$ is not open in the subspace topology on $h(T)$. But from the definition of the enhanced order topology we see that this set is not open in the enhanced order topology on $h(T)$ either. On the other hand, the set $\{W \in h(T): W \geq h(x)\}$ is open in the enhanced order topology and open in the subspace topology on $h(T)$.

Let $v \in \mathbb{R}$ with $v>g(y)$. By Lemma 2 there is a $u \in g\left(T_{2}\right)$ with $u>g(y)$ such that

$$
\left\{w \in T_{2}: g(w)<u\right\} \subset\left\{w \in T_{2}: g(w)<v\right\} .
$$

Hence in $\mathbb{R}_{0}$,

$$
\{W \in h(T): W<(u, 0)\} \subset\{W \in h(T): W<(v, 0)\},
$$

where the right member is open in the subspace topology on $h(T)$ and the left member is open in both the enhanced order topology and the subspace topology on $h(T)$.

(iii) $x \in T$ has neither an immediate successor nor an immediate predecessor in $T$, and $x$ is neither the greatest nor the least element of $T$. It follows that $x \in T_{2}$ and $h(x)=(g(x), 0)$. Now $(g(x), 1) \in \mathbb{R}_{0}$, so the set $\{W \in h(T): W \leq h(x)\}$ is open in the subspace topology on $h(T)$. By definition, this set is also open in the enhanced order topology on $h(T)$.

Take any $v^{\prime} \in \mathbb{R}$ with $v^{\prime}<g(x)$. It follows from Lemma $2^{\prime}$ that there exists a $u^{\prime} \in g(T)$ such that $u^{\prime}<g(x)$ and

$$
\left\{w \in T_{2}: g(w)>u^{\prime}\right\} \subset\left\{w \in T_{2}: g(w)>v^{\prime}\right\} .
$$

It follows that

$$
\left\{W \in h(T): W>\left(u^{\prime}, 0\right)\right\} \subset\left\{W \in h(T): W>\left(v^{\prime}, 0\right)\right\},
$$

where the right member is open in the subspace topology on $h(T)$, and the left member is open in both the subspace topology and the enhanced order topology on $h(T)$.

(iv) $x \in T$ has an immediate successor $y \in T$ but no immediate predecessor in $T$, and $T$ has neither a greatest nor a least element. It follows that $x \in T_{2}$ and $h(x)=(g(x), 0)$. Now $(g(x), 1) \in \mathbb{R}_{0}$, so the set $\{W \in h(T): W \leq h(x)\}$ 
is open in the subspace topology on $h(T)$, and is also open in the enhanced order topology on $h(T)$.

Take any $v^{\prime} \in \mathbb{R}$ with $v^{\prime}<g(x)$. Just as in the argument for case (iii) we find a $u^{\prime} \in g(T)$ such that $u^{\prime}<g(x)$ and

$$
\left\{W \in h(T): W>\left(u^{\prime}, 0\right)\right\} \subset\left\{W \in h(T): W>\left(v^{\prime}, 0\right)\right\},
$$

where the right member is open in the subspace topology on $h(T)$ and the left member is open in both the subspace topology and the enhanced order topology on $h(T)$.

Let $x \in T$ and $h(x)$ lie in a subbasic open set of one of the forms $\{W \in$ $h(T): W>A\},\{W \in h(T): W<A\},\{W \in h(T): W \leq A\}$ in the enhanced order topology on $h(T)$. Then it follows from (i), (ii), (iii), (iv) that this set contains an open set in the subspace topology on $h(T)$ containing $h(x)$. Thus if $T$ has no greatest or least element, every open set in the enhanced order topology on $h(T)$ is also open in the subspace topology on $h(T)$. Analogous arguments using (i), (ii), (iii), (iv) show that if $T$ has no greatest or least element, every open set in the subspace topology on $h(T)$ is also open in the enhanced order topology on $h(T)$. We can relax the assumption that $T$ have no greatest or least element by adjoining to $T$ a copy of the ordered set of rational numbers, $\mathbb{Q}$, all of whose elements exceed all the elements of $T$ and a copy of all $\mathbb{Q}$ all of whose elements are exceeded by all elements of $T$. Finally, the subspace topology and the enhanced order topology coincide on $h(T)$, and (1) $\Rightarrow(2)$.

From $(1) \Rightarrow(2)$ we deduce that $(1) \Rightarrow(3)$ and $(1) \Rightarrow(4)$. From Lemma 4 we deduce that $(2) \Rightarrow(1)$ and $(4) \Rightarrow(1)$. From Lemma 4 we also deduce that $(3) \Rightarrow(5)$. Finally, the enhanced order topology contains the order topology, so it follows that $(5) \Rightarrow(1)$.

If each element of $T$ is an immediate successor or predecessor, then the enhanced order topology coincides with the order topology on $h(T)$. For such order spaces we deduce the following from Theorem III.

Corollary 1. Let $T$ be an order space in which each element is an immediate successor or predecessor. Then $T$ is separable if and only if $T$ is homeomorphic to a subspace of $\mathbb{R}_{0}$.

From Theorems I, II and III we obtain:

Corollary 2. Let $S_{0}$ be a (nonvoid) subset of $\mathbb{R}_{0}$, let $S_{1}$ be a (nonvoid) subset of $\mathbb{R}$ and let $S_{2}$ be a (nonvoid) subset of $\mathbb{Q}$. Then the enhanced order space $S_{0}^{*}$ is homeomorphic to a subspace of $\mathbb{R}_{0}$, the order space $S_{1}$ is homeomorphic to a subspace of $\mathbb{R}$, and the order space $S_{2}$ is homeomorphic to a subspace of $Q$.

We leave the proofs of Corollaries 1 and 2. 


\section{References}

[K] J. Kelley, General topology, van Nostrand, New York, 1955.

[S] W. Sierpinski, General topology, The University of Toronto Press, 1934. 
F. S. CATER 